\title{
Measurement on Axial Reaction Turbine Stage
}

\author{
Marek Klimko ${ }^{1, *}$, Pavel Žitek ${ }^{1}$, and Richard Lenhard ${ }^{2}$ \\ ${ }^{1}$ University of West Bohemia in Pilsen, Univerzitni 8, Pilsen, 30100, Czech Republic \\ ${ }^{2}$ University of Žilina, Department of Power Engineering, Univerzitná 8215/1, 010 26, Žilina, Slovak \\ republic
}

\begin{abstract}
This article describes a measuring methods and evaluating measured data on a single-stage axial turbine with reaction $(\sim 50 \%)$. One turbine operating mode was selected, in which the traversing behind the nozzle and bucket with two 5 -hole pneumatic probes took place. The results are distributions of flow angles, reactions, or losses distribution/efficiencies along the blades.
\end{abstract}

\section{Introduction}

The experimental device (VT-400) is a 1:2 scale model of the high-pressure steam turbine part (Figure 1). The working medium is air sucked from the atmosphere by a compressor. The turbine stage is located at the compressor suction. The maximum pressure drop that the compressor can achieve is $12.5 \mathrm{kPa}$. The dynamometer measures RPM and torque. The mass flow of the working medium is determined by a standardized nozzle. Static pressure taps are located in individual sections of the turbine, and two 5-hole pneumatic probes are used for detailed flow field measurement.

More information about experimental device can be found in the papers [2, 3].
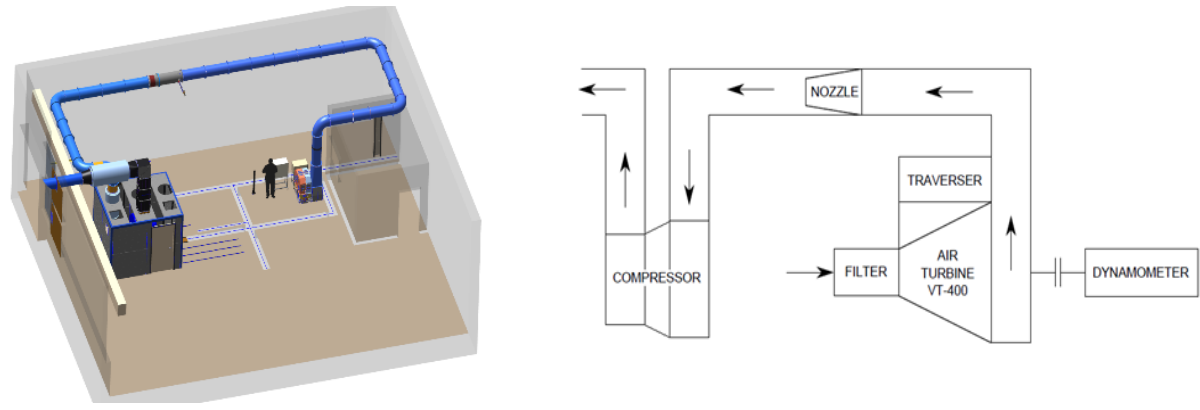

Fig. 1. Schematic representation of the test rig.

\footnotetext{
* Corresponding author: klimko@kke.zcu.cz
} 
At present, the department has three turbine stages with different reactions, which were designed by our industrial partner Doosan Skoda Power. Their basic geometric parameters are listed in the following table (Table 1 - the currently measured stage is marked in red).

Table 1. Geometrical parameters of tested stages.

\begin{tabular}{|c|c|c|c|c|c|}
\hline \multicolumn{3}{|l|}{ Nozzle } & \multicolumn{3}{|c|}{ Bucket } \\
\hline Root diameter [mm] & $D_{P}^{N Z L}$ & 400 & Root diameter [mm] & $D_{P}{ }^{B C K}$ & 400 \\
\hline Blade length [mm] & $L^{N Z L}$ & 45.5 & Blade length [mm] & $L^{B C K}$ & 47 \\
\hline Chord [mm] & $c^{N Z L}$ & 22.5 & \multicolumn{3}{|c|}{ Output relative velocity angles $\left[{ }^{\circ}\right]$} \\
\hline $\begin{array}{l}\text { Output absolute velocity } \\
\text { angle }\left[{ }^{\circ}\right]\end{array}$ & $\alpha_{1}$ & $14.5^{\circ}$ & $\begin{array}{l}\text { Low reaction - LR } \\
(\sim 25 \%)\end{array}$ & $\beta_{2}{ }^{L R}$ & $19.9^{\circ}$ \\
\hline & & & $\begin{array}{c}\text { Mid reaction - MR } \\
(\sim 35 \%)\end{array}$ & $\beta_{2}{ }^{M R}$ & $19^{\circ}$ \\
\hline & & & $\begin{array}{c}\text { Full reaction - FR } \\
(\sim 50 \%)\end{array}$ & $\beta_{2}{ }^{F R}$ & $14.5^{\circ}$ \\
\hline
\end{tabular}

\section{Measurement and data evaluation}

We perform two types of measurements on the turbine. The aim of the first type is to determine the basic turbine stage characteristic, i.e. the dependence of the circumferential efficiency on the speed ratio $u / c_{f}$ (ratio of circumferential velocity and fictive (isentropic) velocity. In our case, the modes $\left(u / c_{f}\right)$ are set by regulating the compressor (RPM), while the pressure drop on the turbine changes. From the measurement point of view only static pressures in individual sections of the flow path (see Figure 2), input/output static pressures of the mass nozzle, data from the dynamometer (RPM and torque), stage output temperature and the mass nozzle output temperature are measured.

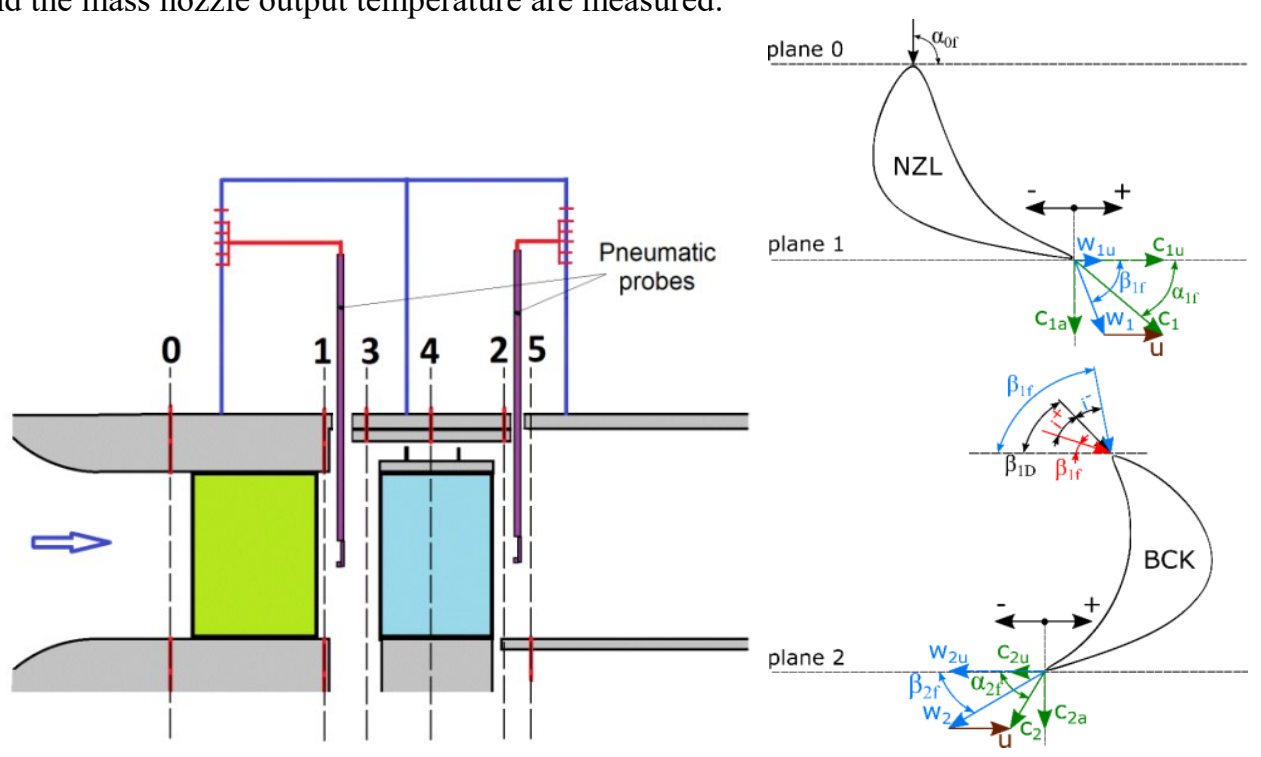

Fig. 2. Turbine stage measured planes (left) and velocity/angle orientation (right). 
The second type of measurement is used for detailed flow fields measurement in the wake behind the nozzle and bucket. From these data it is possible to evaluate the radial distribution of losses, reaction, flow angles, Mach and Reynolds numbers. In this case, a constant mode is set at the beginning of the measurement $\left(u / c_{f}=\right.$ const. $)$. In this article we will deal with the second type of measurement.

Both probes must be calibrated before operation. The calibration was realized by setting a certain angle $\Theta$, at which the pressures are measured for different values of the angle $\varphi$ in the range $\varphi=\left(-20^{\circ} ; 20^{\circ}\right)$. Subsequently, the turning device is shifted by an angle $\Theta$ and the pressures are measured again for the entire range of angles $\varphi$. The range of angles $\Theta$ was also $\Theta=\left(-20^{\circ} ; 20^{\circ}\right)$. Calibration coefficients (two pressure, two angular) were calculated from the obtained data.

$$
\begin{gathered}
C_{S T A T}=\frac{p_{1}-p_{s}}{p_{1}-p_{m}} ; C_{T O T}=\frac{p_{1}-p_{t}}{p_{1}-p_{m}} ; C_{\varphi}=\frac{p_{2}-p_{3}}{p_{1}-p_{m}} ; C_{\theta} \\
=\frac{p_{4}-p_{5}}{p_{1}-p_{m}}
\end{gathered}
$$

The calibration coefficients with the probe rotation angles together create data for $3 \mathrm{D}$ graphs (Figure 3). Mathematical interpretation of these 3D surfaces in the form of regression equations roots matrix is one of the main inputs in the evaluation process from wake traversing behind the nozzle and bucket.
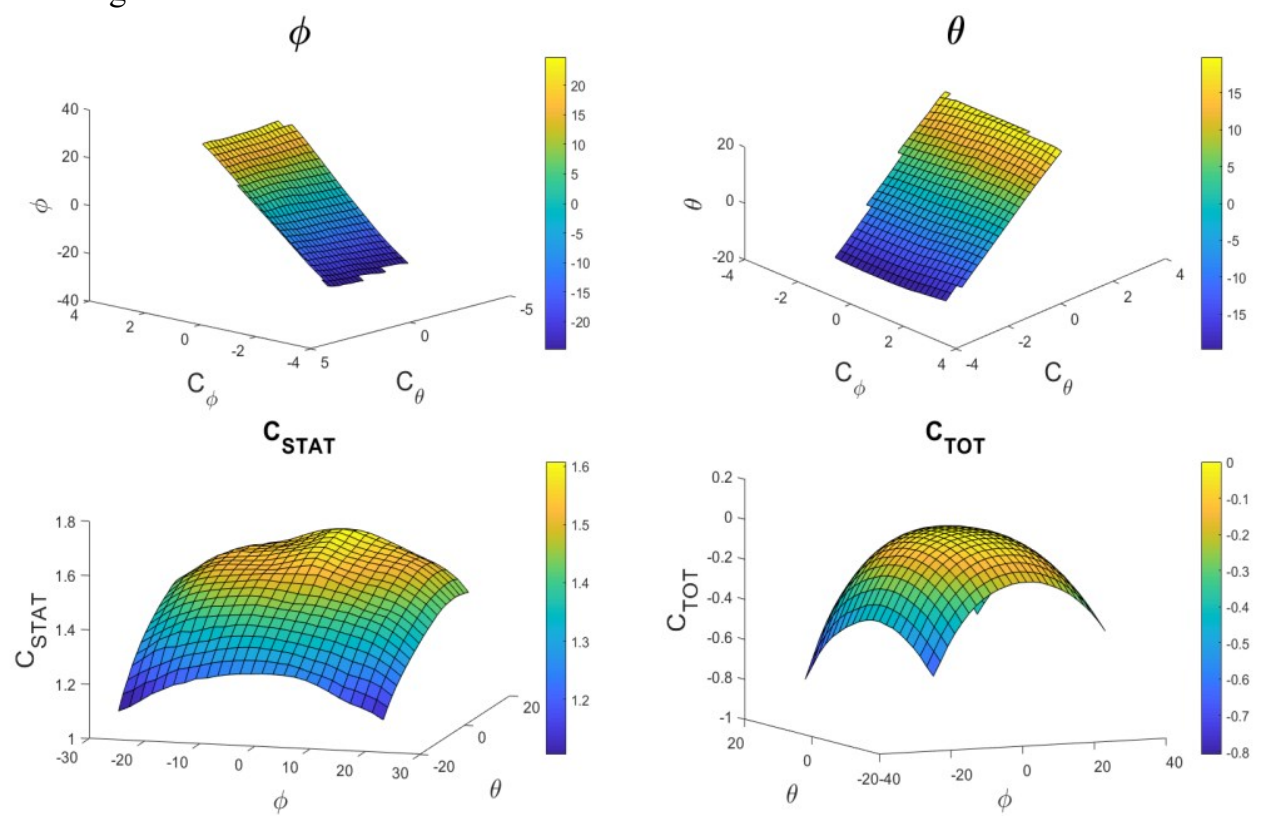

Fig. 3. 3D Graphs for fist 5-hole probe.

\section{Data evaluation procedure}

SW developed at the Department of Power System Engineering is used to evaluate data from the turbine. The SW was programmed in the "Matlab ${ }^{\circledR}-$ App Designer" interface. 
The program enables area averaging or averaging weighted by mass flow (in the case of incompressible flow by axial velocity only).

In the evaluation process, only the axial velocity is averaged over the area. Other parameters are averaged by mass flow over a multiple of the blade pitch. The area averaging method has a great weakness in the wake areas, where, for example, losses increase disproportionately. In mass averaging, the flow through these areas will be taken into account and the obtained values are therefore much closer to reality.

\section{Results}

The flow field measurement was preceded by the turbine mode choice. The turbine mode was selected, which corresponds to an output flow angle of $\sim 90^{\circ}$ (approximately axial output). The turbine speed in this mode was 2000 RPM and the pressure drop per stage corresponded to $2425 \mathrm{~Pa}$. According to the design data, the turbine should operate close to the optimal operating mode. The evaluated angles $\beta_{1 f}$ and $\beta_{2 f}$ compared with the design values correspond to this (see Figure 4).
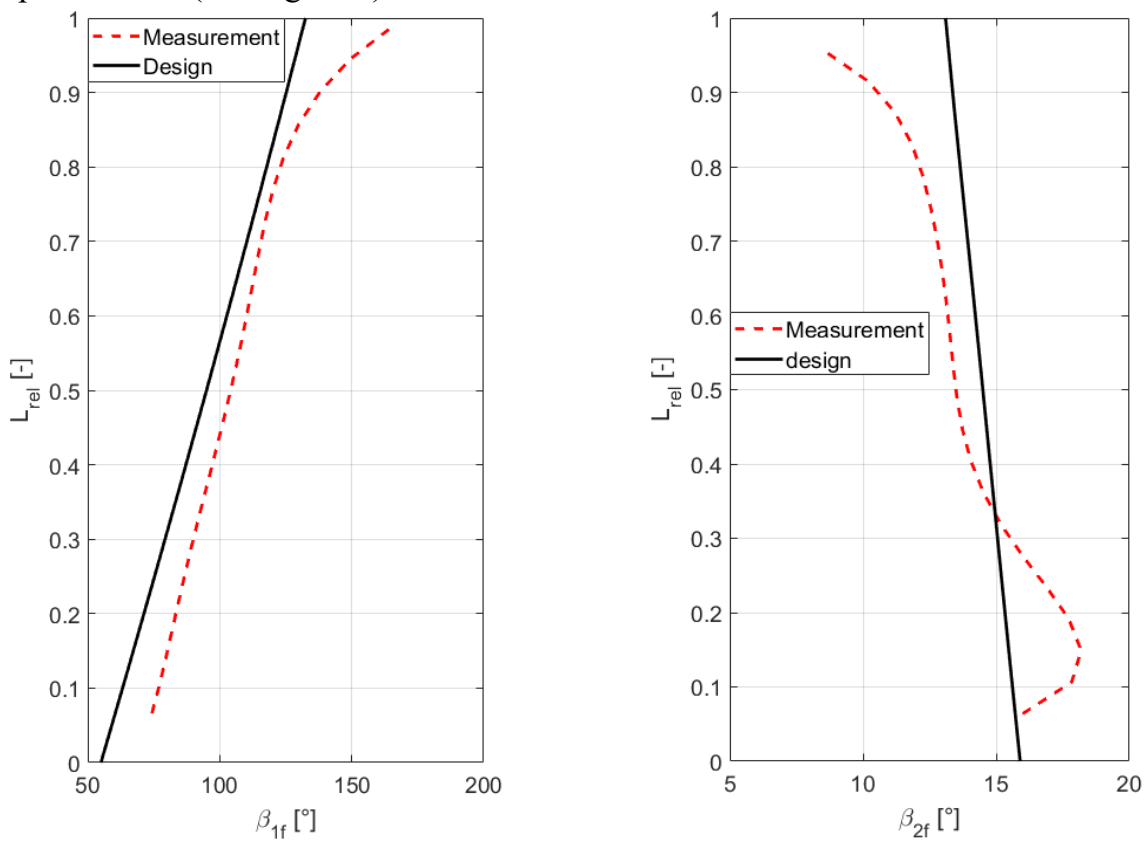

Fig. 4. Bucket input relative velocity flow angle radial distribution (left) bucket output relative velocity flow angle radial distribution (right).

Dependence on Figure 5 (left) describe the radial distributions of the individual efficiencies. Nozzle efficiency distribution is relatively constant along the blade. A significant decrease in nozzle efficiency occurs in the blade tip area, where the inlet cavity influence into the shroud sealing is very pronounced. This trend in the bucket tip area can also be observed on bucket efficiency distribution where, on the contrary, the parasitic flow from the shroud sealing interact with main flow, which result in a local losses increase.

Now let's take a closer look at the turbine stage efficiency. As mentioned above, the efficiency is evaluated from the individual points of the expansion line. The theory presents several relations, which can also be used to calculate the values of the circumferential stage efficiency. This is for example literature [5], where the relation for the 
circumferential efficiency is directly a function of the reaction $\rho$, the output absolute velocity flow angle from the nozzle $\alpha_{1 f}$, the output relative velocity flow angle from the bucket $\beta_{2 f}$ and the velocity ratios $\mathrm{u} / \mathrm{c}_{\mathrm{f}}, \chi=\frac{c_{1}}{c_{f}}, \psi=\frac{w_{2}}{w_{2, i z}}$.

$$
\eta_{S T}^{\text {Theory }}=f\left(\rho, \alpha_{1 f}, \beta_{2 f}, \frac{u}{c_{f}}, \chi, \psi\right)
$$

Subsequent modifications can be used to obtain the resulting relationship for circumferential efficiency:

$$
\begin{aligned}
\eta_{S T}^{\text {Theory }}= & 2 \frac{u}{c_{f}}[\psi \\
& \cdot \cos \beta_{2} \\
& \cdot \sqrt{\rho-\chi^{2}(\rho-1)+\left(\frac{u}{c_{f}}\right)^{2}-2 \chi \frac{u}{c_{f}} \cos \alpha_{1} \sqrt{1-\rho}}- \\
& \frac{-\frac{u}{c_{f}}+\chi \cdot \cos \alpha_{1} \sqrt{1-\rho}}{2}
\end{aligned}
$$

The following dependence (Figure 5 right) shows a relatively good agreement between the evaluated radial distributions. In addition, the dependence of turbine stage efficiency from 1D simulation [4] is plotted in the graph, too. The calculation was performed for the root, mid and tip blade profile and the boundary conditions were chosen in accordance with the conditions during the measurement. It is obvious that in the root and tip areas it is not possible to expect agreement with measurement, because the 1D calculation does not capture the effects of secondary flow arising from the interaction of parasitic flow from shroud sealing with the main flow or the endwall effects at the blade root.
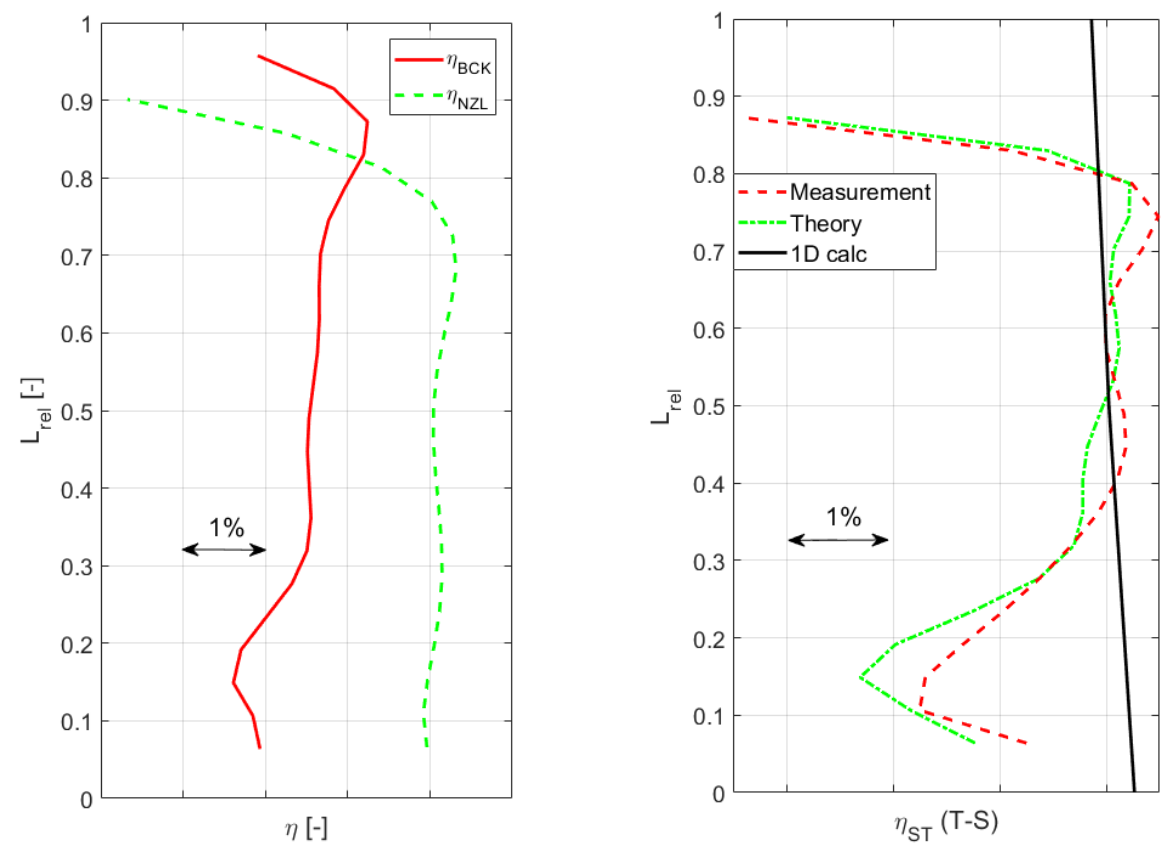
Fig. 5. Comparison of nozzle and bucket efficiency radial distribution (left), comparison of stage efficiencies radial distribution (right).

\section{Conclusion}

The article deals with measurements on an air turbine of the Department of Power System Engineering. The above results are evaluated from measurements using two pneumatic 5hole probes for one specific turbine operating mode. Further activities will focus on the study of the different turbine operating modes. These modes can be achieved by regulating the compressor, i.e. setting different pressure drop to the turbine stage, resulting in stage load changes, velocity triangles redistribution, etc. After measuring current stage with $\sim 50 \%$ reaction, the same measurements will follow for the other two reactions $(\sim 35 \%$ and $\sim 25 \%)$. These measurements could provide interesting comparisons in the individual stages behavior under different operating conditions.

Acknowledgment: The presented work was financially supported by the Ministry of Education, Youth and Sport Czech Republic Project LQ1603 (Research for SUSEN). This work has been realized within the SUSEN Project (established in the framework of the European Regional Development Fund (ERDF) in project CZ.1.05/2.1.00/03.0108). This work was founded by projects KEGA 063ŽU-4/2018.

\section{Nomenclatore}

$c$ - Absolute velocity

$p_{c}$ - Absolute pressure

$p_{m}$ - Averaged pressure $p_{m}=\left(p_{2}+\right.$

$\left.p_{3}+p_{4}+p_{5}\right) / 4$

$p_{s}$ - Static pressure

$S$ - Area

$T$ - Absolute temperature

$u$ - Circumferential velocity

$w$ - Relative velocity

$\alpha_{1}$ - Output absolute velocity flow angle from nozzle $\alpha_{2}$ - Output absolute velocity flow angle from bucket

$\beta_{1}$ - Output relative velocity flow angle from nozzle

$\beta_{2}$ - Output relative velocity flow angle from bucket

$\eta_{S T}$ - Turbine stage efficiency

$\theta$ - Pitch probe angle

$\rho$ - Degree of reaction

$\varphi$ - Yaw probe angle

$\psi$ - Velocity ratio $\psi=\frac{w_{2}}{w_{2, i z}}$

$\chi$ - Velocity ratio $\chi=\frac{c_{1}}{c_{f}}$

\section{References}

1. N. A. Cumpsty, J. H. Horlock, Averaging Non-Uniform Flow for a Purpose, Paper No. GT2005-68081. DOI: 10.1115/GT2005-68081 (2008)

2. M. Klimko, D. Okresa, Measurements in the VT 400 Air Turbine, Acta Polytechnica, 56(2), pp. 118 (2016) DOI: 10.14311/AP.2016.56.0118

3. M. Klimko, D. Okresa, Experimental Research of Reaction Blading on Air Turbine VT400, EPJ Web of Conference, 114 (2016) DOI: 10.1051/epjconf/201611402055

4. M. Klimko, Theoretical Study of Turbine Stage Characteristics in Off-Design Conditions, AIP Conference Proc., 2047 (2018) DOI: 10.1063/1.5081641

5. A. V. Scegljajev, Parni turbiny 1., SNTL Moscow, pp. 145 (1983) 
6. T. Yasa, G. Paniagua, Robust Procedure for Multi-Hole Probe Data Processing, Flow Measurement and Instrumentation, 26, pp. 46-54 (2012) DOI: 10.1016/j.flow measinst.2012.03.004 